استعمال معامل الاقتران المتعدد لتمييز القصائد العربيت لعينتمن الشعراء

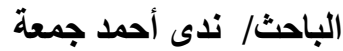

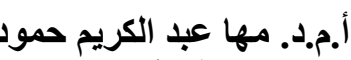

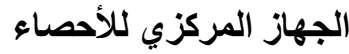

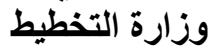

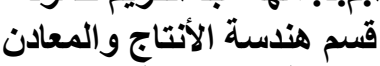

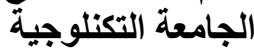

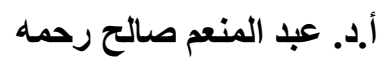

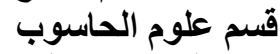

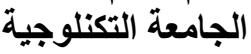
maha_alrawi@yahoo.com monem.rahma@yahoo.com nadaalzubaidy@yahoo.com

\title{
تاريخ التقايم:2018/3/28
}

تاريخ القبول:2018/6/6

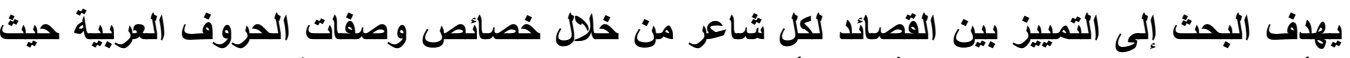

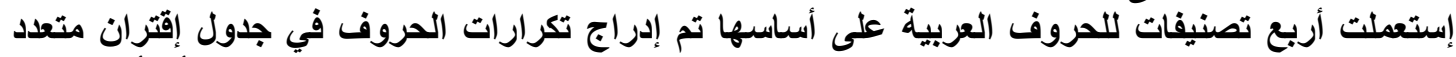

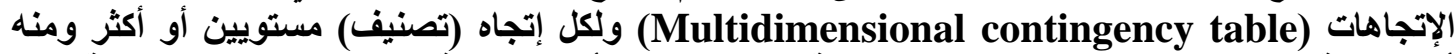
أيجاد قيمة معامل الإقتران لكل قصيدة ضمن عينة البحث والتي تتألف من ستة شعر اءع من عصور مختلفة ولكل شاعر خمس قصائد.

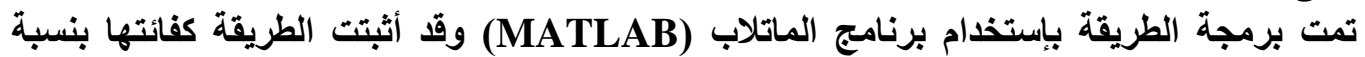

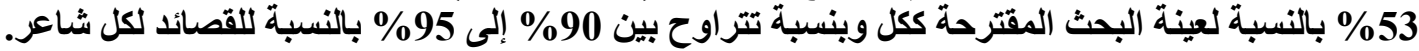

المطالحات الرئيسة لابمث/ الثعر العربي، أثكال الحروف العربية، صفات الحروف العربية، أل التعريف، جدول الإقتران المتعدد، مقاييس الاقتران.

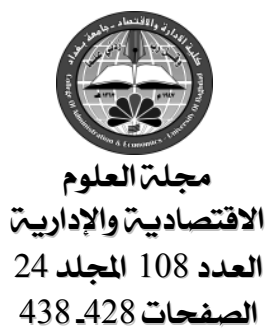


استعمال معامل الاقتران المتعدر لتمييز القصائد العربية لعينة من الشعراء

1. المقدهة (Introduction)

تبرز أهمية هذا البحث في إستخلاص صفات وخصائص الحروف العربية ضمن نص من القصائد

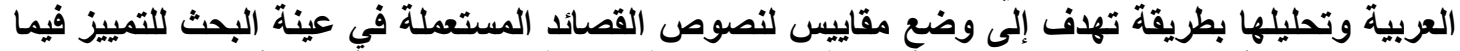

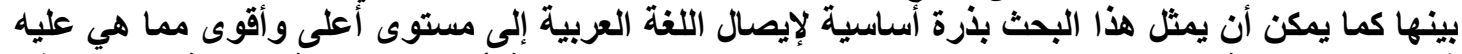

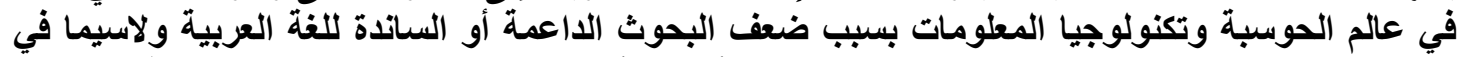

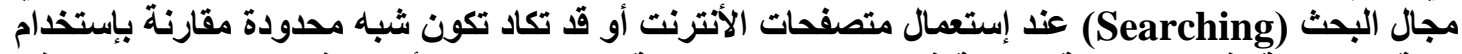

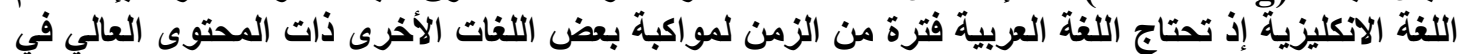
مجال تكنولوجيا المعلومات.

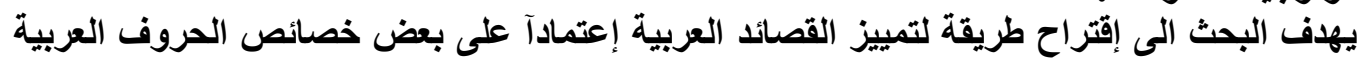

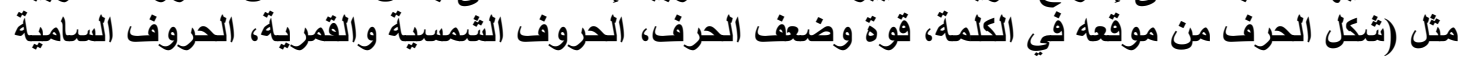

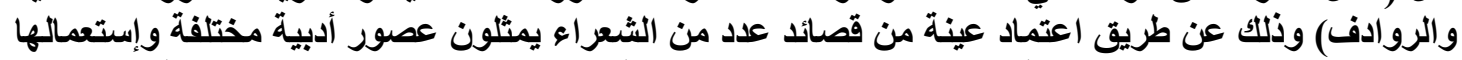

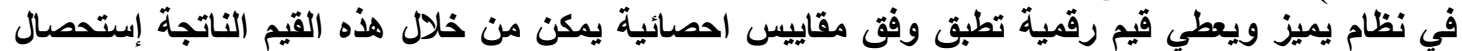
فروقات وتشابهات تمكن من تمييز شاعر معين عن بقية الشعراء وفئ وايضا تعطي نسبة تقارب وتثنابه شعر

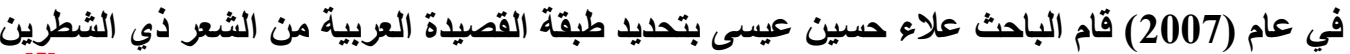
الثـاعر نفسه.

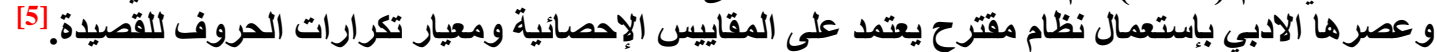

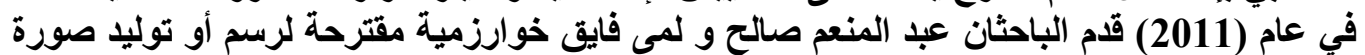

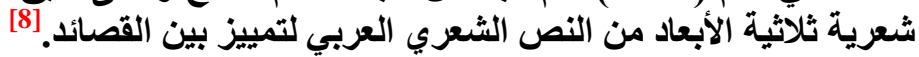

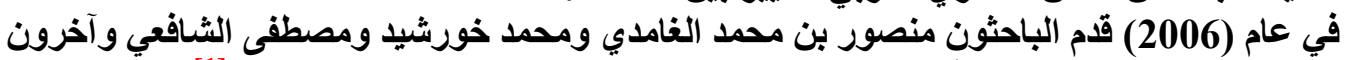

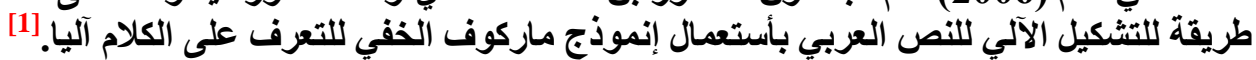

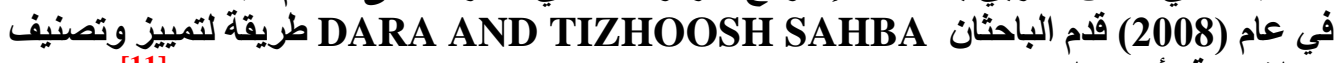

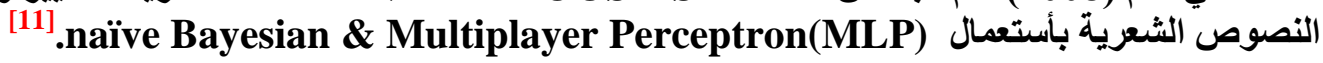

2. تصنيف الحروف العربية (Classification of Arabic letters)

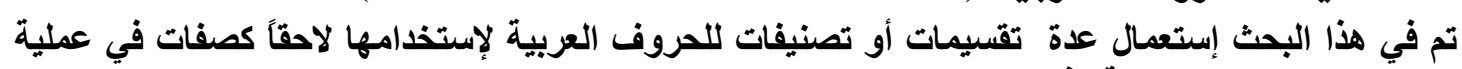
التمييز للنصوص الثعرية إذ تم إستعمال اريع تصانيف للحروف وكل تصنيف بعدات من المستويات وهي لإني

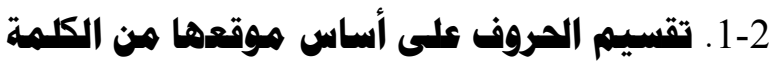

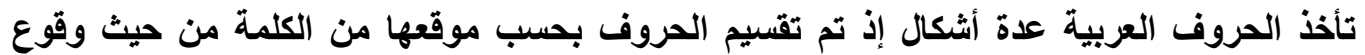

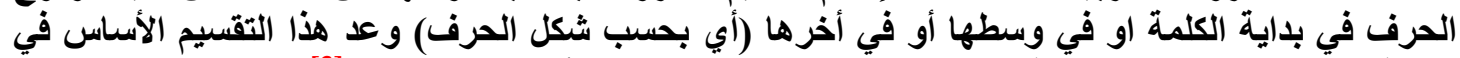

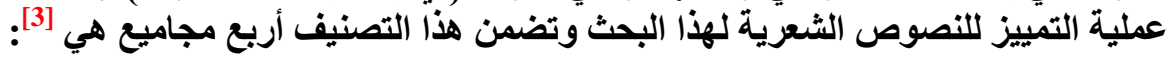

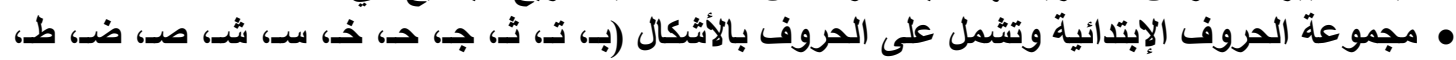

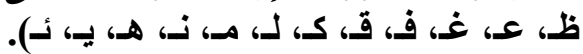
• مجموعة الحروف الوسطية وتثمل على الحروف بالأثكال (ب، تُ، ث، جـ، ح، خ، سد، شـ، صد، ضـ،

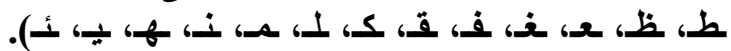

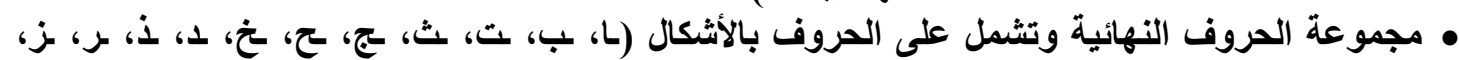

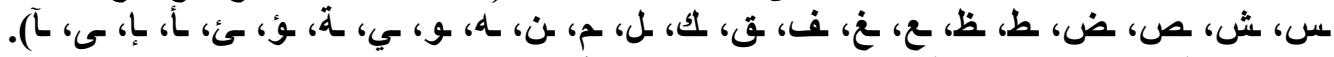

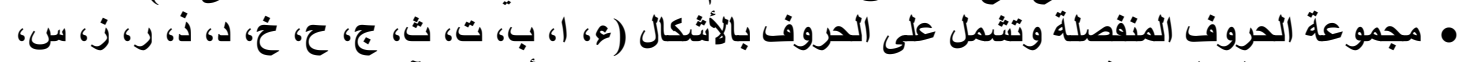

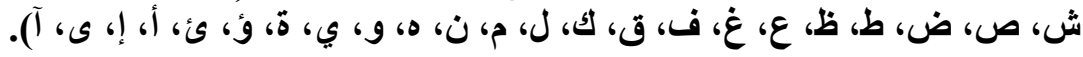




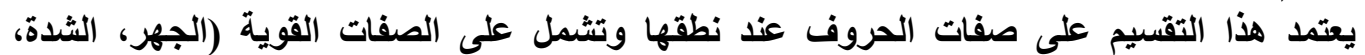

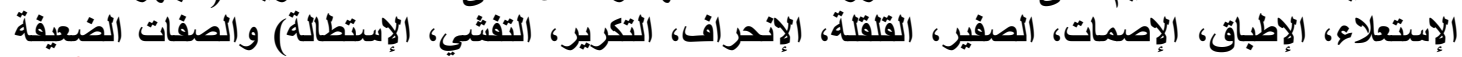

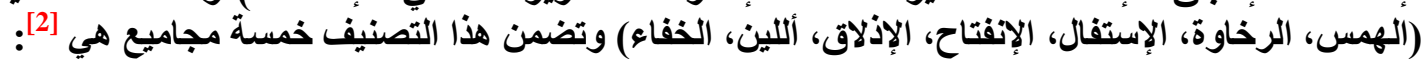

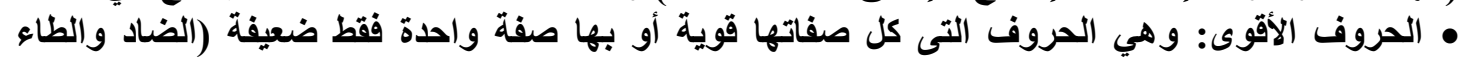
والظاء). • الحروف القوية: وهي الحروف التى صفاتها القوية أكثر من صفاتها الضعيفة (الهمزة والجيم والدال و الغين و الصاد و القاف).

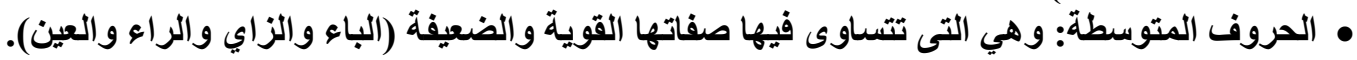

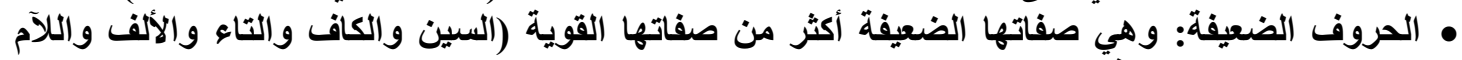

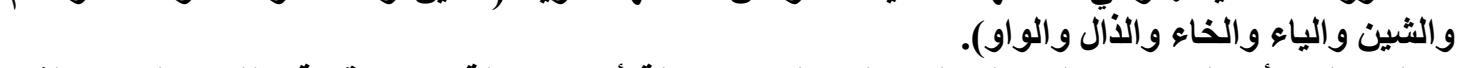
• الحروف الأضعف: وهي الحروف التى كل صفاتها ضعيفة أو بها صفة والحدة قوية (الفاء والحاء والثاء و الهاء والميم والنون).

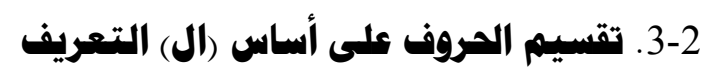

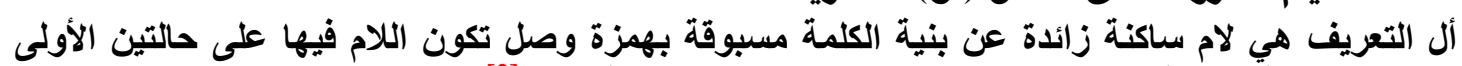

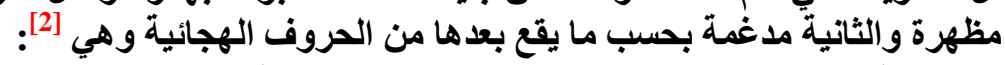

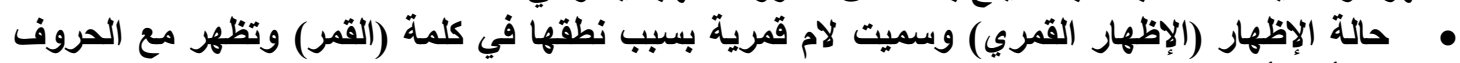

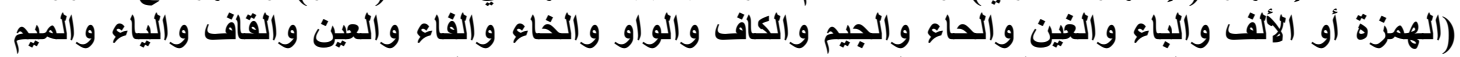

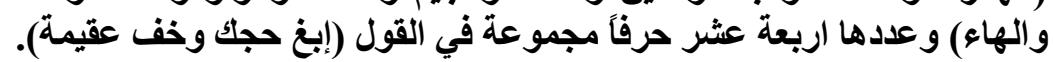

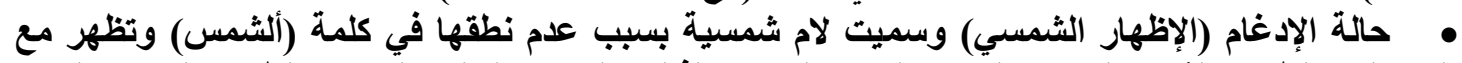

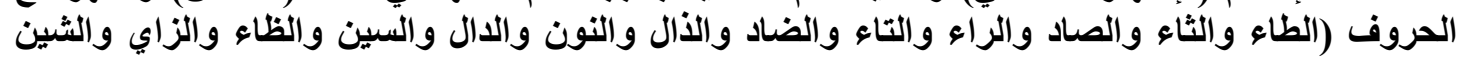

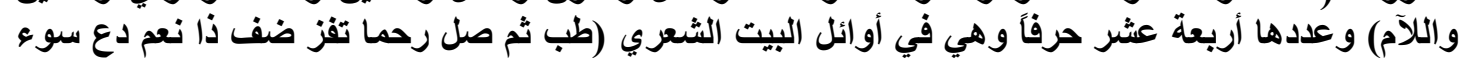
ظن زر شريفا للكرم).

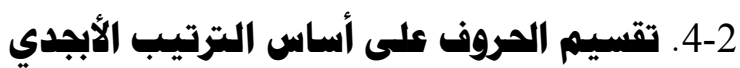

وهو تقسيم منسوب إلى الساميين (أصحاب اللسان العبري) حيث تتثابه حروف اللغتين العبرية والعربية وتزيد

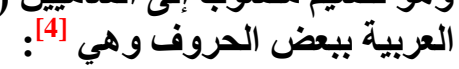

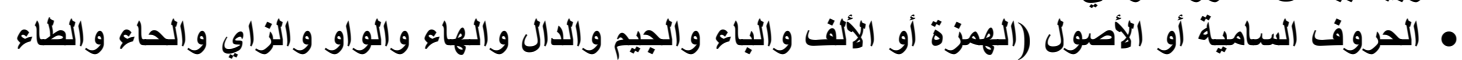

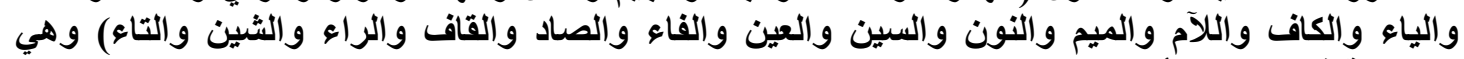

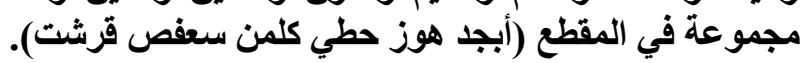

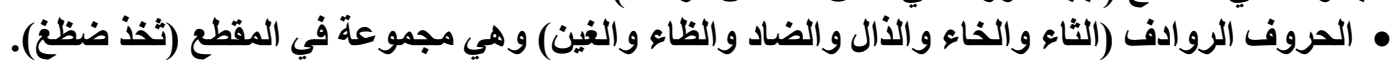

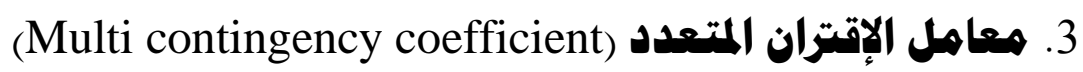

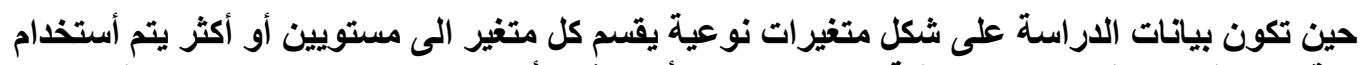

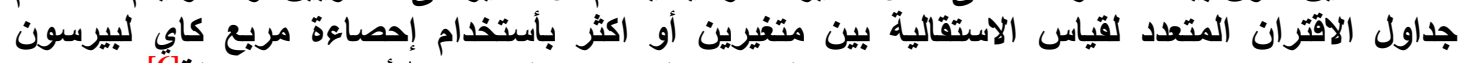
(Pearson chi-squared statistics) تم في هذا البحث إستعمال جدول إقتران مكون من أربع إتجاهات (r)

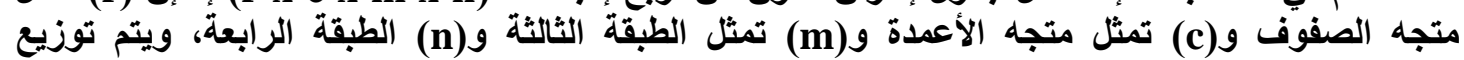

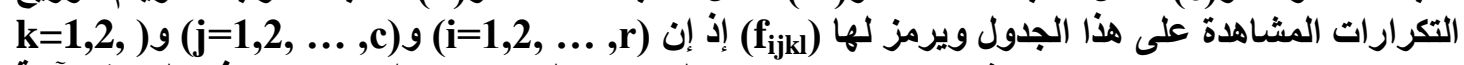

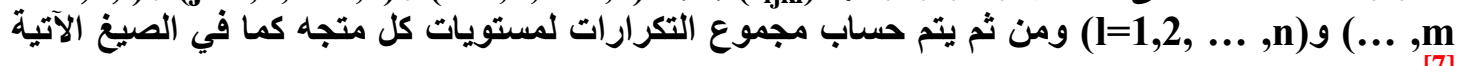




\section{استعمال معامل الاقتران المتعدر لتمييز القصائد العربية لعينة من الشعراء}

$$
\begin{aligned}
& f_{i_{\mathrm{m}}}=\sum_{j=1}^{c} \sum_{k=1}^{m} \sum_{l=1}^{n} f_{i j k l} \\
& f_{. j_{\mathrm{s}}}=\sum_{i=1}^{r} \sum_{k=1}^{m} \sum_{l=1}^{n} f_{i j k l} \\
& f_{\mathrm{sk} .}=\sum_{i=1}^{r} \sum_{j=1}^{c} \sum_{l=1}^{n} f_{i j k l} \\
& f_{\mathrm{m} l}=\sum_{i=1}^{r} \sum_{j=1}^{c} \sum_{k=1}^{m} f_{i j k l}
\end{aligned}
$$

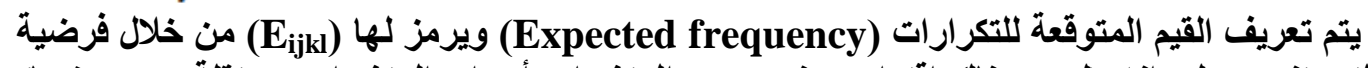

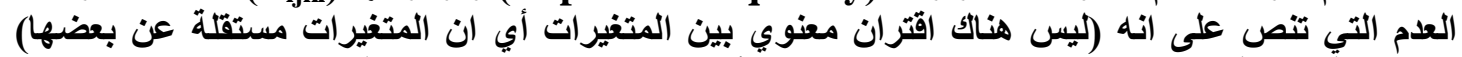

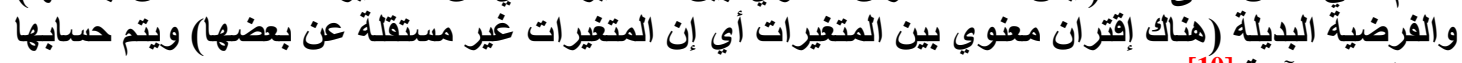

$$
\begin{aligned}
& E_{i j k l}=N \cdot \frac{f_{i_{m}}}{N} \cdot \frac{f_{j_{n}}}{N} \cdot \frac{f_{k_{k}}}{N} \cdot \frac{f_{\mathrm{m} l}}{N} \\
& E_{i j k l}=\frac{f_{i_{\mathrm{m}}} \cdot f_{. j \mathrm{n}} \cdot f_{\mathrm{s} k_{\mathrm{n}}} \cdot f_{\mathrm{m} l}}{N^{3}}
\end{aligned}
$$

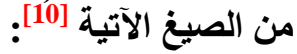

ومن ثم يتم إحتساب إحصاءة مربع كاي لبيرسون (Pearson chi-squared statistic) ويرمز لها (X) كما في الصيغة الآتية [9]: $X^{2}=\sum_{\substack{i \\ \text { s }}} \sum_{j} \sum_{k} \sum_{\substack{l \\ \text { (degree }}} \frac{\left(f_{i j k l}-E_{i j k l}\right)^{2}}{E_{i j k l}}$

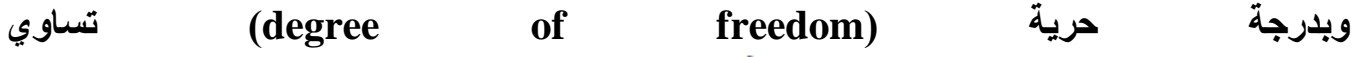

$$
\begin{aligned}
& \text {. (df }=\text { r.c.k.l } l-r-c-k-l+3)
\end{aligned}
$$

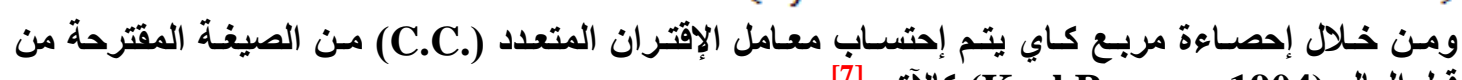

$$
\text { C.C. }=\sqrt{\frac{X^{2}}{X^{2}+N}}
$$

قبل العالم (Karl Pearson 1904) كالآتي [7]

تتراوح قيمة معامل الاقتران بين (1,0) فإذاكان قريب من الصفر دل ذلك على عدم وجود أقتران وإذا كان واحد دل ذلك على وجود علاقة بين المتغيرات المدروسة. 


\section{استعمال معامل الاقتران المتعرد لتمييز القمائد العربية لعينة من الشعراء}

4. التطبيق العملي (Practical application)

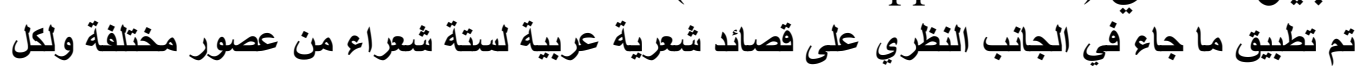

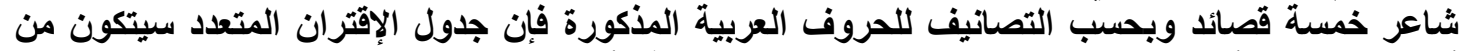

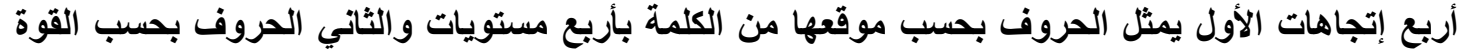

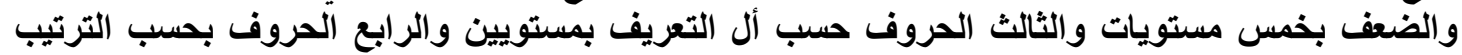

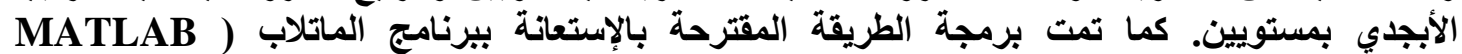
(R2014a ) وبحسب الخطوات الآتية:

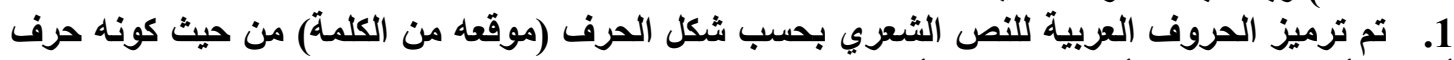

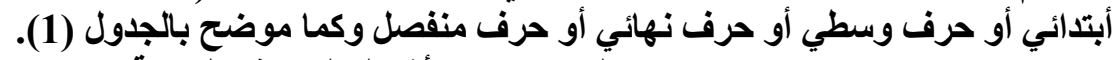
جدول (1) ترميز أشكال الحروف العربية.

\begin{tabular}{|c|c|c|c|c|c|c|c|c|c|}
\hline No & $\begin{array}{c}\text { Letter } \\
\mathrm{X} \\
\end{array}$ & $\begin{array}{c}\text { First } \\
\text { Letter }\end{array}$ & $\mathbf{R 1}$ & $\begin{array}{c}\text { Middle } \\
\text { Letter }\end{array}$ & $\mathbf{R} 2$ & $\begin{array}{c}\text { Final } \\
\text { Letter }\end{array}$ & $\mathbf{R 3}$ & $\begin{array}{l}\text { Separate } \\
\text { d Letter }\end{array}$ & R4 \\
\hline 1 & 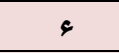 & - & - & - & - & - & - & 8 & D11 \\
\hline 2 & 1 & - & - & - & - & $L$ & C12 & 1 & D12 \\
\hline 3 & ب & بـ & $\mathbf{A 1 3}$ & $\div$ & B13 & 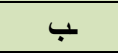 & C13 & ب & D13 \\
\hline 4 & $ت$ & ت & A14 & 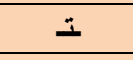 & B14 & $ت$ & C14 & $ت$ & D14 \\
\hline 5 & $\dot{H}$ & $\dot{3}$ & A15 & $\dot{\dot{A}}$ & B15 & $\dot{H}$ & C15 & $\dot{ث}$ & D15 \\
\hline 6 & ج & $\rightarrow$ & A16 & ج & B16 & ج & C16 & ج & D16 \\
\hline 7 & $\tau$ & $\boldsymbol{A}$ & A17 & $\sim$ & B17 & $\tau$ & C17 & $\tau$ & D17 \\
\hline 8 & $\dot{\tau}$ & $\dot{\Delta}$ & A18 & $\dot{-}$ & B18 & $\dot{\tau}$ & C18 & $\dot{\tau}$ & D18 \\
\hline 9 & 1 & - & - & - & - & \pm & C19 & $د$ & D19 \\
\hline 10 & $j$ & - & - & - & - & $i$ & $\mathrm{C20}$ & $j$ & D20 \\
\hline 11 & J & - & - & - & - & j & C21 & 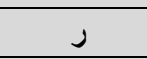 & D21 \\
\hline 12 & $j$ & - & - & - & - & $j$ & $\mathrm{C22}$ & $j$ & D22 \\
\hline 13 & س & سد & $\mathbf{A 2 3}$ & سב & B23 & س & $\mathrm{C23}$ & س & D23 \\
\hline 14 & ش & شـ & A24 & شـ & B24 & ش & C24 & ش & D24 \\
\hline 15 & ص & صد & A25 & صد & B25 & ص & $\mathrm{C25}$ & ص & D25 \\
\hline 16 & ض & ضد & A26 & ضـ & B26 & ض & $\mathrm{C26}$ & ض & D26 \\
\hline 17 & $b$ & $b$ & A27 & b & B27 & $b$ & C27 & $b$ & D27 \\
\hline 18 & ظ & ظ & A28 & ظ & B28 & ظ & C28 & ط & D28 \\
\hline 19 & $\varepsilon$ & ع & A29 & 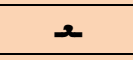 & B29 & $\varepsilon$ & $\mathbf{C 2 9}$ & $\varepsilon$ & D29 \\
\hline 20 & $\dot{\varepsilon}$ & غ & A30 & $\dot{1}$ & B30 & $\dot{\varepsilon}$ & C30 & $\dot{\varepsilon}$ & D30 \\
\hline 21 & ف & $\dot{9}$ & A31 & $\dot{1}$ & B31 & ف & C31 & ف & D31 \\
\hline 22 & ق & ق & A32 & ت & B32 & ق & C32 & ق & D32 \\
\hline 23 & ك & $s$ & A33 & $s$ & B33 & st & C33 & ك & D33 \\
\hline 24 & $J$ & \lrcorner & A34 & 1 & B34 & $\downarrow$ & C34 & $J$ & D34 \\
\hline 25 & P & مـ & A35 & مـ & B35 & $r$ & $\mathbf{C 3 5}$ & P & D35 \\
\hline 26 & ن & ذ & A36 & $\dot{-}$ & B36 & ن & C36 & ن & D36 \\
\hline 27 & 0 & هـ & A37 & $t$ & B37 & $d$ & C37 & 0 & D37 \\
\hline 28 & 9 & - & - & - & - & 9 & C38 & 9 & D38 \\
\hline
\end{tabular}




\section{استعمال معامل اللقتران المتعدر لتمييز القمائد العربية لعينة من الشعراء}

\begin{tabular}{|c|c|c|c|c|c|c|c|c|c|}
\hline 29 & ي & - & A39 & $\div$ & B39 & ي & C39 & ي & D39 \\
\hline 30 & $\ddot{0}$ & - & - & - & - & $\ddot{\alpha}$ & $\mathrm{C} 40$ & $\ddot{0}$ & D40 \\
\hline 31 & ؤ & - & - & - & - & ؤ & C41 & ؤ & D41 \\
\hline 32 & ئ & ــــ & A42 & 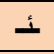 & B42 & سئ & $\mathrm{C42}$ & ئ & D42 \\
\hline 33 & $i$ & - & - & - & - & $\dot{L}$ & $\mathrm{C43}$ & $i$ & D43 \\
\hline 34 & $!$ & - & - & - & - & L & C44 & $!$ & D44 \\
\hline 35 & s & - & - & - & - & $\checkmark$ & $\mathrm{C45}$ & ى & D45 \\
\hline 36 & i & - & - & - & - & $\tau$ & C46 & $\tau$ & D46 \\
\hline
\end{tabular}

2. تم ترميز النص الشعري العربي ومثالاً على ذلك القصيدة الشعرية لأبو العلاء المعري الموضحة بالشكل

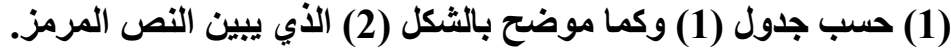
شكل (1) قصيدة شعرية لأبو العلاء المعري. المرئ

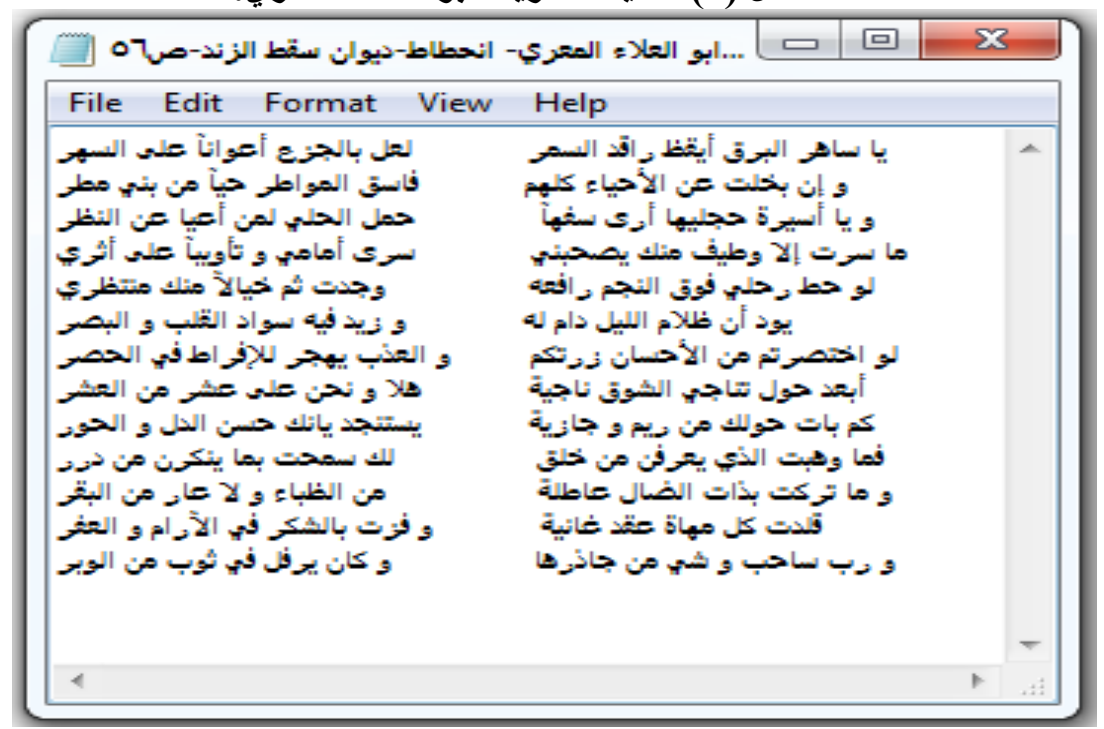

شكل (2) ملف نصي للحروف المرمزة.

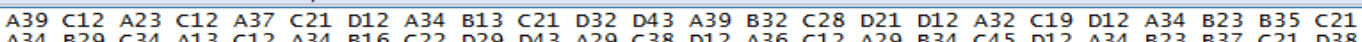

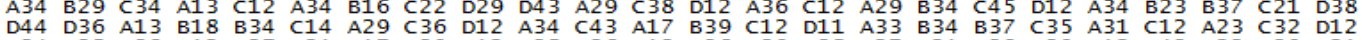

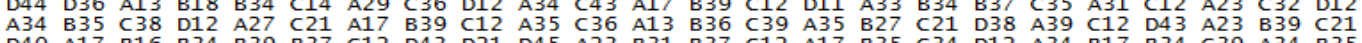

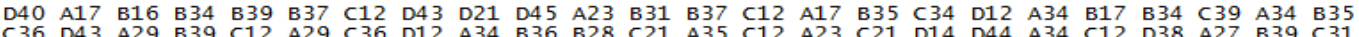

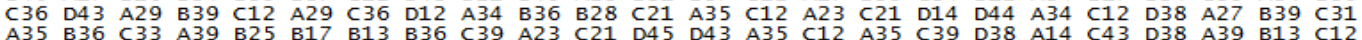

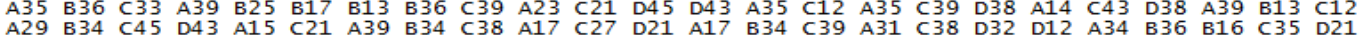

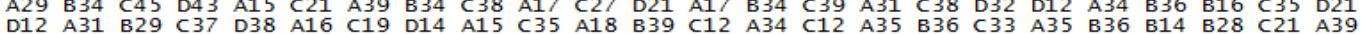

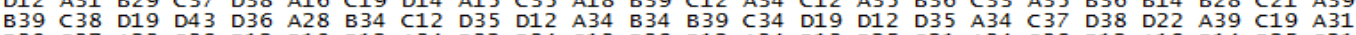

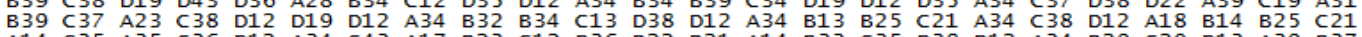

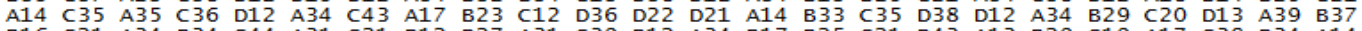

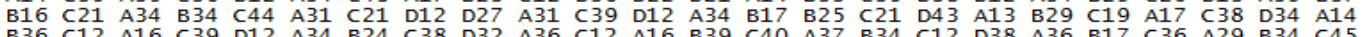
B36 C12 A16 C39 D12 A34 B24 C38 D32 A36 C12 A16 B39 C40 A37 B34 C12 D38 A36 B17 C36 A29 B34 C45

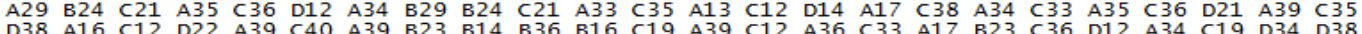

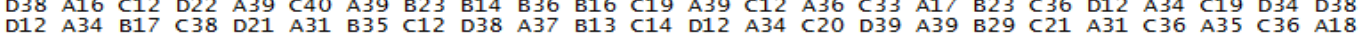

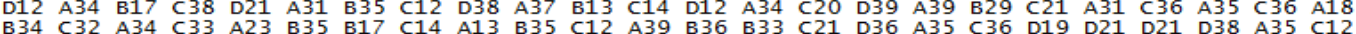

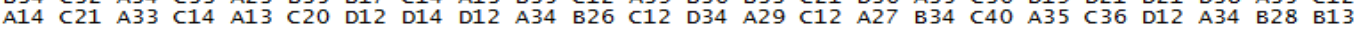

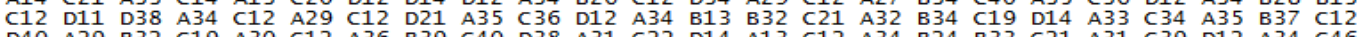

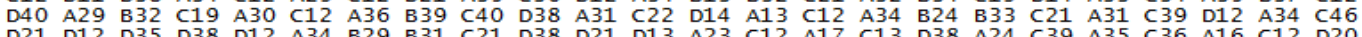

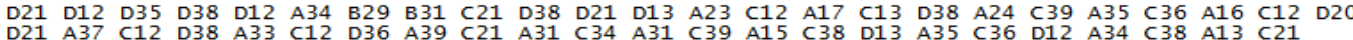




\section{استعمال معامل الاقتران المتعرد لتمييز القمائد العربية لعينة من الشعراء}

3. حساب التكرارات للحروف بحسب التصنيفات الموضحة سابقا للحروف العربية ولكل مستوى.

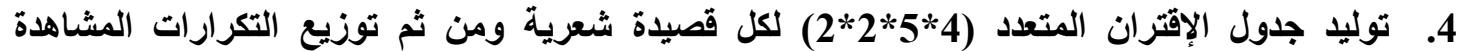

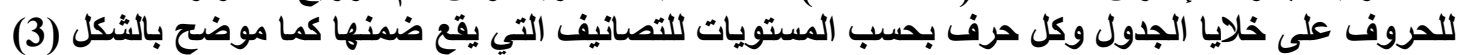

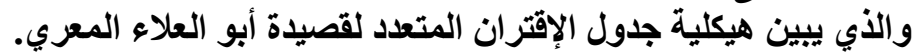

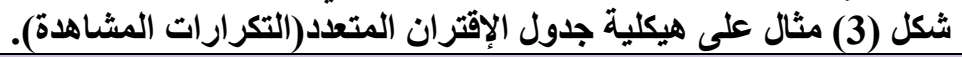

\begin{tabular}{|c|c|c|c|c|c|c|c|c|c|c|c|c|}
\hline & & & & & \multicolumn{8}{|c|}{ Multidimensional contingency table $(4 * 5 * 2 * 2)$, poem 1} \\
\hline \multicolumn{7}{|c|}{$(:,:, 1,1)$} & \multicolumn{6}{|c|}{$(:,:, 1,2)$} \\
\hline 104 & \multicolumn{6}{|c|}{1} & \multicolumn{6}{|c|}{2} \\
\hline \multirow{5}{*}{1} & D2 & 1 & 2 & 3 & 4 & 5 & D2 & 1 & 2 & 3 & 4 & 5 \\
\hline & 1 & 3 & $\mathbf{0}$ & $\mathbf{0}$ & 52 & 5 & 1 & $\mathbf{0}$ & 7 & 20 & 21 & 46 \\
\hline & 2 & 1 & 4 & $\mathbf{0}$ & 29 & 10 & 2 & $\mathbf{0}$ & 9 & 14 & 15 & 21 \\
\hline & 3 & 1 & 8 & 29 & 13 & 16 & 3 & $\mathbf{0}$ & 7 & 2 & 71 & 11 \\
\hline & 4 & 1 & 4 & 16 & 11 & 5 & 4 & $\mathbf{0}$ & 16 & 4 & 58 & 3 \\
\hline \multicolumn{7}{|c|}{$(:,:, 2,1)$} & \multicolumn{6}{|c|}{$(:,:, 2,2)$} \\
\hline \multirow{5}{*}{2} & D2 & 1 & 2 & 3 & 4 & 5 & D2 & 1 & 2 & 3 & 4 & 5 \\
\hline & 1 & 1 & $\mathbf{0}$ & $\mathbf{0}$ & $\mathbf{0}$ & 3 & 1 & $\mathbf{0}$ & 1 & $\mathbf{0}$ & 3 & $\mathbf{0}$ \\
\hline & 2 & 4 & $\mathbf{0}$ & 0 & $\mathbf{0}$ & $\mathbf{0}$ & 2 & $\mathbf{0}$ & $\mathbf{0}$ & 0 & 1 & $\mathbf{0}$ \\
\hline & 3 & 1 & 0 & 0 & 3 & $\mathbf{0}$ & 3 & 0 & $\mathbf{0}$ & $\mathbf{0}$ & $\mathbf{0}$ & $\mathbf{0}$ \\
\hline & 4 & $\mathbf{0}$ & $\mathbf{0}$ & $\mathbf{0}$ & 1 & 0 & 4 & $\mathbf{0}$ & $\mathbf{0}$ & $\mathbf{0}$ & $\mathbf{0}$ & $\mathbf{0}$ \\
\hline
\end{tabular}

5. إحتساب قيم التكرارات المتوقعة لجدول الإقتران بحسب الصيغة (6) ولكل قصيدة شعرية كما موضح

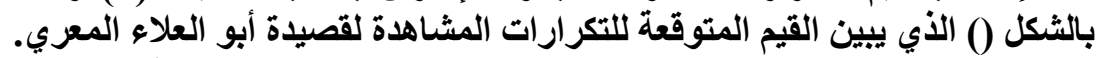
شكل (4) جدول التكرارات المتوقعة.

\begin{tabular}{|c|c|c|c|c|c|c|}
\hline \multicolumn{7}{|c|}{$(:,:, 1,1)$} \\
\hline D4 & & & & & & \\
\hline \multirow{5}{*}{1} & D2 & 1 & 2 & 3 & 4 & 5 \\
\hline & 1 & 1.37 & 6.39 & 9.7 & 31.7 & 13.7 \\
\hline & 2 & 0.91 & 4.26 & 6.46 & 21.1 & 9.13 \\
\hline & 3 & 1.37 & 6.39 & 9.7 & 31.7 & 13.7 \\
\hline & 4 & 1.01 & 4.69 & 7.12 & 23.3 & 10.1 \\
\hline
\end{tabular}

Expected frequency table $(4 * 5 * 2 * 2)$, poem 1

\begin{tabular}{|c|c|c|c|c|c|c|}
\hline \multirow{3}{*}{} & \multicolumn{7}{|c}{$(:,:, 2,1)$} \\
\cline { 2 - 7 } & D1 & 1 & 2 & 3 & 4 & 5 \\
\cline { 2 - 7 } 2 & 1 & 0.05 & 0.22 & 0.33 & 1.07 & 0.46 \\
\cline { 2 - 7 } & 2 & 0.03 & 0.14 & 0.22 & 0.71 & 0.31 \\
\cline { 2 - 7 } & 3 & 0.05 & 0.22 & 0.33 & 1.07 & 0.46 \\
\hline & 4 & 0.03 & 0.16 & 0.24 & 0.79 & 0.34 \\
\hline
\end{tabular}

434

\begin{tabular}{|c|c|c|c|c|c|}
\hline \multicolumn{7}{|c|}{$(:,:, 1,2)$} \\
\hline 12 & 1 & 2 & 3 & 4 & 5 \\
\hline 1 & 2.04 & 9.54 & 14.5 & 47.4 & 20.4 \\
\hline 2 & 1.36 & 6.36 & 9.65 & 31.6 & 13.6 \\
\hline 3 & 2.04 & 9.54 & 14.5 & 47.4 & 20.4 \\
\hline 4 & 1.5 & 7.01 & 10.6 & 34.8 & 15 \\
\hline
\end{tabular}

\begin{tabular}{|c|c|c|c|c|c|}
\hline \multicolumn{7}{|c|}{$(:,:, 2,2)$} \\
\hline D2 & 1 & 2 & 3 & 4 & 5 \\
\hline 1 & 0.07 & 0.32 & 0.49 & 1.6 & 0.69 \\
\hline 2 & 0.05 & 0.21 & 0.33 & 1.07 & 0.46 \\
\hline 3 & 0.07 & 0.32 & 0.49 & 1.6 & 0.69 \\
\hline 4 & 0.05 & 0.24 & 0.36 & 1.17 & 0.51 \\
\hline
\end{tabular}

مجلتمالعلوم الاقتصاديت والإداريت

العدد (108) المجلد (24) لسنت 2018 


\section{استعمال معامل الاقتران المتعرد لتمييز القصائد العربية لعينة من الشعراء}

6. إحتساب قيمة مريع كاي حسب الصيغة (7) ولكل قصيدة حيث تمثّل (N) عداب (2) حروف القصيدة.

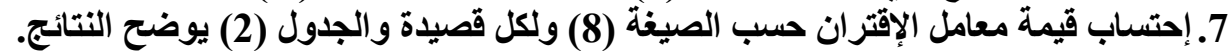

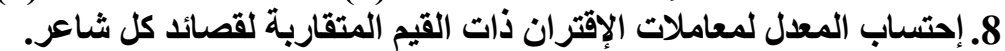

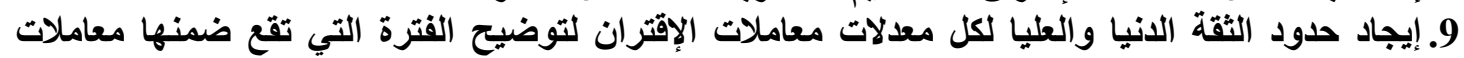

جدول (2) قيم معامل الإقتران

\begin{tabular}{|c|c|c|c|c|c|c|}
\hline الحالة & $\begin{array}{l}\text { الحد الأدنى } \\
\alpha=0.05\end{array}$ & $\begin{array}{c}\text { الحـ الأعلى } \\
\alpha=0.95\end{array}$ & معلال معامل & معامل الإقتران & القصائد & الثاعر \\
\hline نجاح & \multirow{4}{*}{0.764} & \multirow{4}{*}{0.804} & \multirow{4}{*}{0.784} & 0.78 & القصيدة 1 & \multirow{5}{*}{ ابو العلاء المعري } \\
\hline نجاح & & & & 0.79 & القصيدة 2 & \\
\hline نجاح & & & & 0.8 & القصيدة 3 & \\
\hline فشل & & & & 0.77 & القصيدة 4 & \\
\hline فثل & & & & 0.67 & القصيدة 5 & \\
\hline فشل & \multirow{4}{*}{0.701} & \multirow{4}{*}{0.743} & \multirow{4}{*}{0.722} & 0.72 & القصيدة 1 & \multirow{5}{*}{ علي بن أبي طالب } \\
\hline نجاح & & & & 0.7 & القصيدة 2 & \\
\hline فثل & & & & 0.73 & القصيدة 3 & \\
\hline فشل & & & & 0.73 & القصيدة 4 & \\
\hline فشل & & & & 0.85 & القصيدة 5 & \\
\hline نجاح & \multirow{3}{*}{0.803} & \multirow{3}{*}{0.838} & \multirow{3}{*}{0.82} & 0.81 & القصيدة 1 & \multirow{5}{*}{ بدر شاكر السياب } \\
\hline نجاح & & & & 0.82 & القصيدة 2 & \\
\hline نجاح & & & & 0.83 & القصيدة 3 & \\
\hline فثل & & & & 0.72 & القصيدة 4 & \\
\hline فثل & & & & 0.72 & القصيدة 5 & \\
\hline فشل & \multirow{5}{*}{0.715} & \multirow{5}{*}{0.747} & \multirow{5}{*}{0.731} & 0.72 & القصيدة 1 & \multirow{5}{*}{ حاتم الطائي } \\
\hline فشل & & & & 0.72 & القصيدة 2 & \\
\hline فثل & & & & 0.73 & القصيدة 3 & \\
\hline فشل & & & & 0.75 & القصيدة 4 & \\
\hline فشل & & & & 0.74 & القصيدة 5 & \\
\hline نجاح & \multirow{4}{*}{0.68} & \multirow{4}{*}{0.694} & \multirow{4}{*}{0.687} & 0.69 & القصيدة 1 & \multirow{5}{*}{ كعب بن زهير } \\
\hline نجاح & & & & 0.68 & القصيدة 2 & \\
\hline نجاح & & & & 0.69 & القصيدة 3 & \\
\hline نجاح & & & & 0.69 & القصيدة 4 & \\
\hline فثل & \multirow{6}{*}{0.745} & & & 0.79 & القصيدة 5 & \\
\hline نجاح & & \multirow{5}{*}{0.779} & \multirow{5}{*}{0.762} & 0.75 & القصيدة 1 & \multirow{5}{*}{ صفي الدين الطلي } \\
\hline نجاح & & & & 0.75 & القصيدة 2 & \\
\hline نجاح & & & & 0.77 & القصيدة 3 & \\
\hline نجاح & & & & 0.75 & القصيدة 4 & \\
\hline نجاح & & & & 0.78 & القصيدة 5 & \\
\hline
\end{tabular}




\section{استعمالا معاملا الاقتتران المتعدر لتمييز القمائد العربية لعينة من الشعراء}

ومن خلال إحتساب الكفاعة لكل شاعر (الكفاءة تساوي عدد القصائد ذات معامل إقتران متجانس مقسوم

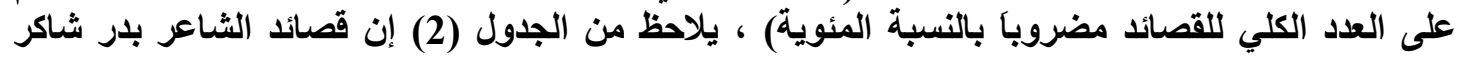

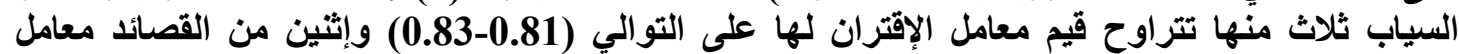

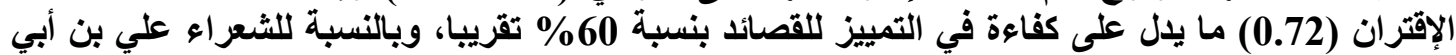

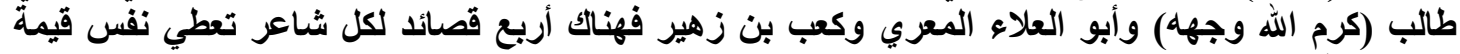

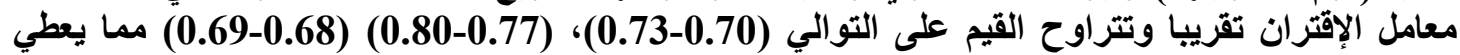

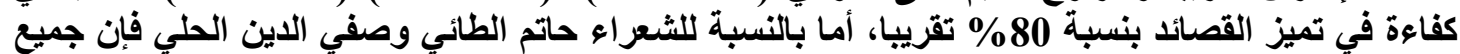

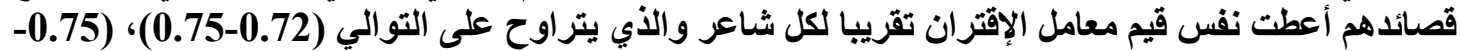

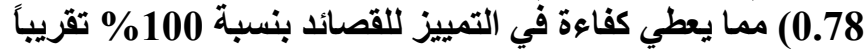

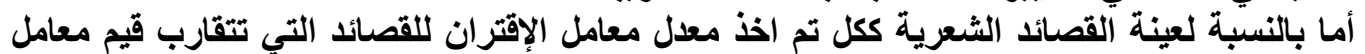

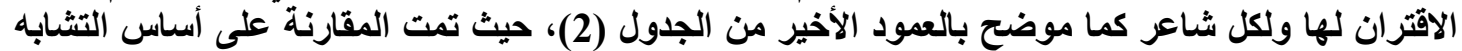

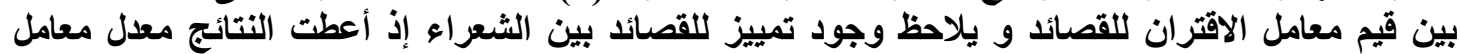

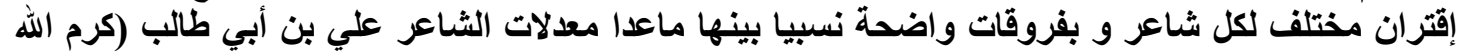

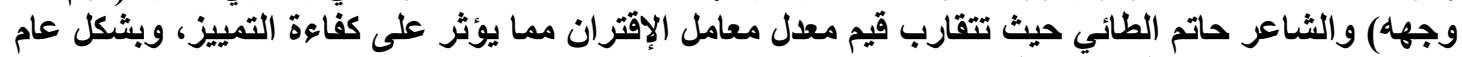

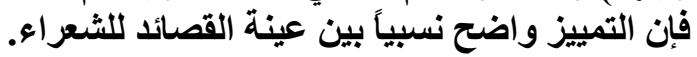

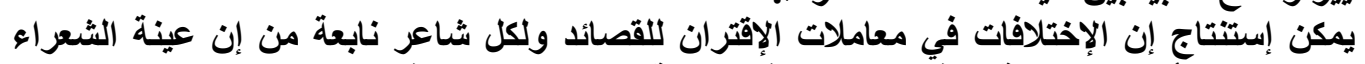

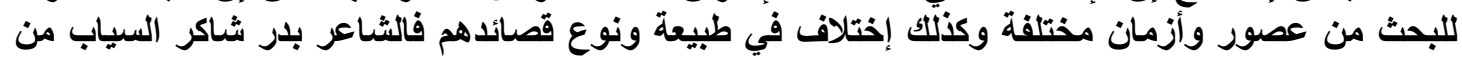

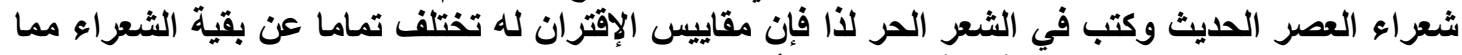

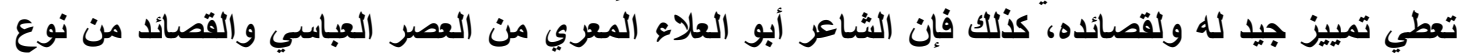

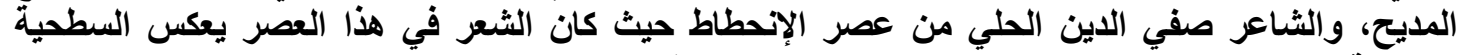

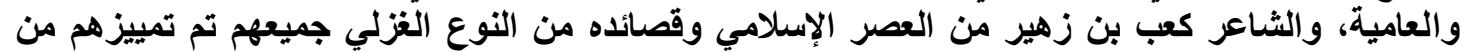
خلال قيم مختلفة لمقاييس الإقتران.

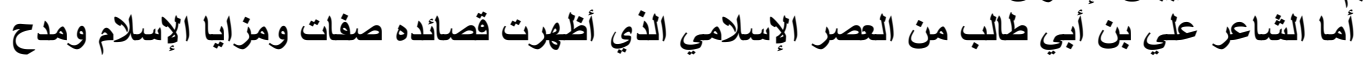

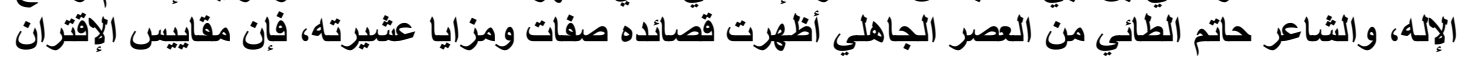

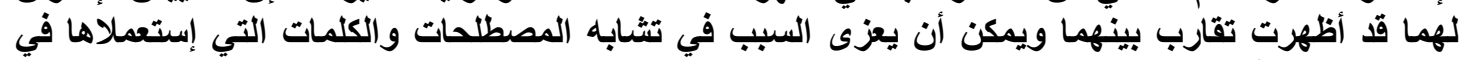
قصائد هم للعناية نفسها.

\section{5. الإستنتاجات (Conclusion) :}

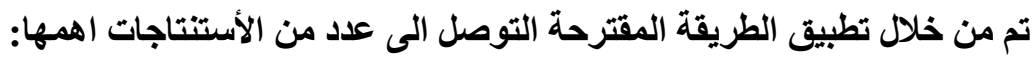

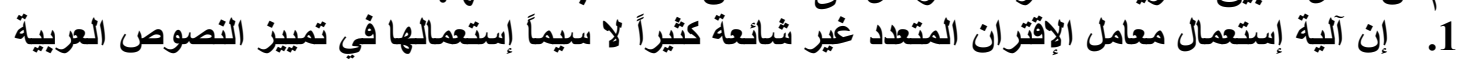

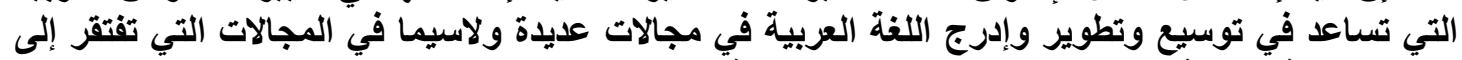

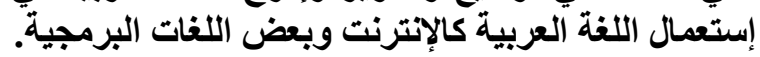
2.

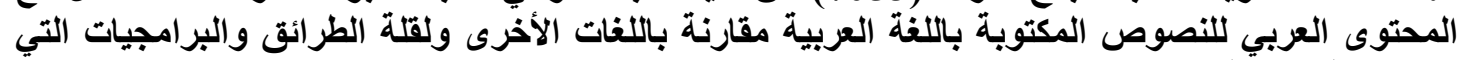

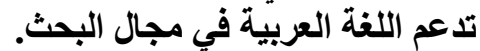

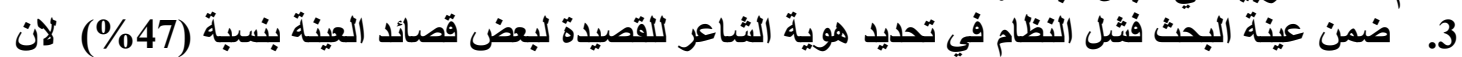

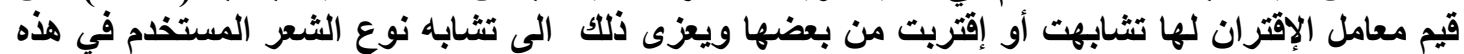

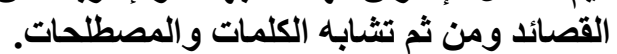




\section{استعمال معامل الاقتران المتعرد لتمييز القمائد العربية لعينة من الشعراء}

\section{المادر (References)}

1. الغامدي ، منصور ،خورشيا ، محمد ، الثافعي ، مصطفى ، " نظام حاسوبي لتثكيل النص العربي " ، 2006

2. المرصفي، عبد الفتاح السيد عجمي، 1980، "هداية القاري إلى تجويد كلام الباري"، الطبعة الثانية،

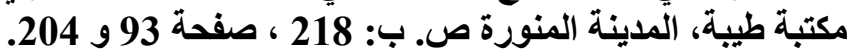

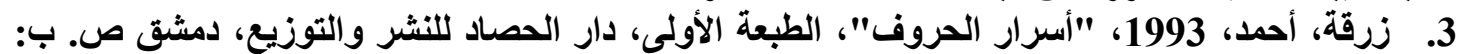
113. 4490

4. صالح، فخري محمد، 1994، "اللغفة العربية أداء ونطقا وإملاء وكتابة"، الطبعة الثانية، دار الوفاء، المنصورة: ص. ب:

5.

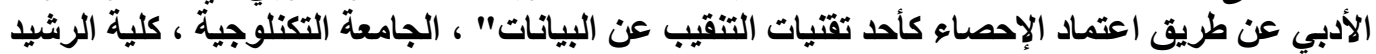

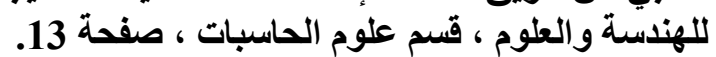

6. Chowdhury, Sadeq R. and Mcgilchrist, C. A., 2001, "Analysis of contingency tables with clustered observation", Aust. N. Z. J. Stat. 43(3), 351-358, page 3.

7. Everitt, B. S., 1977, "The analysis of contingency tables", Halsted press, a division of john Wiley \& sons, inc., newyork, pages $64 \& 68$.

8. Rahma , Abd Al_Monem, Faik, Luma, 2011, "Implementation Of 3D Virtual Poetry Image By Using Bezier Cubic Splines And Open GL Primitives" , Eng.\&Tech.Journal, Vol.29, No.12, page 2364.

9. Shaffer, Juliet Oopper, 1973, "Defining and testing hypotheses in multidimensional contingency tables", Psychological Bulletin, vol. 79, no. 2, 127141 , page 6.

10. Stemmler, Mark, 2014, "Person-centered methods", Springer Cham Heidelberg newyork Dordrecht London, page 5.

11. TIZHOOSH, SAHBA, AND DARA, 2008 , " Poetic Features for Poem Recognition: A Comparative Study ", JOURNAL OF PATTERN RECOGNITION RESEARCH (2008) 24-39, page 27. 
استعمال معامل الاقتران المتعدر لتمييز القصائد العربية لعينة من الشعراء

\section{Using Multidimensional Contingency Coefficient to Discrimination Arabic Poems for Sample of Poets}

\section{Abstract}

The purpose of this paper to discriminate between the poetic poems of each poet depending on the characteristics and attribute of the Arabic letters. Four categories used for the Arabic letters, letters frequency have been included in a multidimensional contingency table and each dimension has two or more levels, then contingency coefficient calculated.

The paper sample consists of six poets from different historical ages, and each poet has five poems. The method was programmed using the MATLAB program, the efficiency of the proposed method is $53 \%$ for the whole sample, and between $90 \%$ and $95 \%$ for each poet's poems.

Keywords: Arabic poetry, Arabic letters shapes, Arabic letters characteristics, Al-alta'reef, multidimensional contingency table, contingency measurements. 